\title{
Adaptarse o Morir supervivencia de entidades económicas en mercados municipales. Caso Mercado Gómez Palacio, Durango ${ }^{4}$
}

\author{
Hortensia Hernández Vela 1 \\ Fátima del Rocío Betancourt Conde 2 \\ Saúl Enrique González Rodríguez 3 \\ Luis Alonso González Roldán 4
}

\begin{abstract}
Resumen
Gran parte de los mercados municipales han desaparecido como resultado del cambio en el entorno a los que no se han podido adaptar, pero algunos de ellos como el mercado Gómez Palacio de Durango aún sobrevive como población organizacional en donde la competencia es una fortaleza y porque sus locatarios han podido cambiar, no solamente los productos sino inclusive su actividad o giro. En esta investigación bajo el enfoque de población organizacional, se analiza el contexto de este mercado, las estrategias que han implementado sus locatarios para sobrevivir, describiendo también algunas de las condiciones del negocio y sus demandas.
\end{abstract}

Palabras clave: Adaptación, poblaciones organizacionales, estrategias.

\begin{abstract}
Almost all typical local markets in Mexico have disappeared, they can't respond to environment changes, only a few could adapt to it, someone like Gómez Palacio Market in Durango survives like an ecological organization, where the competition is a strength. The members of this community have changed not only products but also business activity. This work analyzes under theoretical framework of Organizational Ecology, which are the strategies implemented by little stand owners to survive, describe also business condition and demands.
\end{abstract}

Palabras clave: Adaptation, ecological organization, strategies.

Se reconoce la participación y colaboración para la realización de la presente investigación a los participantes del VII Verano de Investigación de la FECA-UJED: Fátima del Rocío Betancourt Conde, Saúl Enrique González Rodríguez y Luis Alonso González Roldán, Juvenal Casas Duarte, Carlos Fernando Álvarez Sosa, Jorge Alberto Mancinas, Edith García, Vanessa Pablos Esquivel, Diana Karina Soto Martínez, Carlos Ignacio Díaz Zamora y Luis Ávila Flores y de los recursos para apoyo de esta investigación de Programa Integral de Fortalecimiento Institucional PIFI-2015.

\footnotetext{
1Hortensia Hernández. Vela Investigador de la Universidad Juárez del Estado de Durango y miembro de la Red Administración y Gestión en las Organizaciones, correo: paloma@ujed.mx

2 Fátima del Rocío Betancourt Conde egresada de la Licenciatura en Economía de la Universidad Juárez del Estado de Durango participante del VII verano de Investigación, correo: fatybetancourt@gmail.com

3 Saúl Enrique González Rodríguez egresado de la Licenciatura en Economía de la Universidad Juárez del Estado de Durango participante del VII verano de Investigación, correo: miro_04@live.com.mx

4 Luis Alonso González Roldan egresado de la Licenciatura en Economía de la Universidad Juárez del Estado de Durango participante del VII verano de Investigación, correo: keanu_7@hotmail.com
} 


\section{Introducción}

Un mercado tradicional es un recinto en el que se venden artículos de primera necesidad, generalmente alimenticios. Los mercados están formados por puestos individuales que ofrecen productos de diversa índole con predominancia en los productos frescos, tales como carnes, frutas, verduras, pescado, etc. Se instalan en el centro de los barrios o localidades.

Los mercados están llenos de cultura e historia; muestran a lo largo del tiempo la evolución mercantil y la modificación que han sufrido en ciudades donde se encuentran. El consumo de la gente cambia y el mercado se adapta. Para los duranguenses el Mercado Gómez Palacio es un sitio emblemático y lleno de folclor. (Lastra, 2013)

El inmueble nació en la época del Porfiriato, siendo gobernador Francisco Gómez Palacio y Bravo), de ahí el nombre del Mercado. La construcción inició con cinco edificios, pero sólo se terminaron tres; el nuevo mercado y el terreno elegido para la edificación fue en el convento de San Antonio (Manzana entre Pasteur y Patoni, 20 de Noviembre y 5 de Febrero), precisamente donde había estado la huerta de dicho convento; el Palacio Municipal y la nueva penitenciaría; los edificios inconclusos fueron el hospital y el teatro.

El mercado "Gómez Palacio" fue fundado en 1882 por el gobemador del Estado de Durango, Francisco Gómez Palacio y Bravo; dicho mercado se encuentra ubicado en el Centro Histórico de la ciudad de Durango, y comprende la manzana ubicada en avenida 20 de noviembre, calle Pasteur, Patoni y 5 de febrero. El mercado se conforma por distintas áreas, los cuales se dedican a la comercialización de diversos tipos de productos, como lo son las frutas, talabartería, yerbería, carnicería, floristería, comida típica, suvenires, entre otras cosas. (Rosales, 2013)
El mercado ha sufrido grandes transformaciones en los últimos años. La llegada de los supermercados ha provocado la desaparición de los mercados en la ciudad de Durango, con excepción del Mercado "Gómez Palacio". Este mercado pasó de sólo ofrecer víveres, y a lo largo del tiempo ha ido cambiando los productos que se comercializan y los giros de los locales.

En los mercados se ve con claridad lo que es la competencia, ya que existen dentro del mismo gran cantidad de locales que ofrecen los mismos productos, dando así la oportunidad a los visitantes de elegir al que sea de su mayor conveniencia, pero a la vez es esta una de sus mayores fortalezas de ahí que se les considere como poblaciones organizacionales.

La llegada de los supermercados ha provocado la desaparición de los mercados en la ciudad de Durango, que no lograron adaptarse con excepción del Mercado "Gómez Palacio", de ahí que nuestra investigación tiene por objetivo establecer que el mercado es una unidad económica constituida por micronegocios que han sobrevivido como resultado de un proceso de adaptación.

Para establecer el marco de la investigación se basa en lo que se considera un micronegocio (unidad económica) y la teoría de la ecología organizacional en relación a los micronegocio se puede considerar que aunque el desempeño de los micronegocios no es suficiente, representa una gran parte de la economía nacional su falta de eficiencia está determinada por muy diversas causas. Sociodemográficas sobre todo por variables económicas y de mercado: rama de actividad, región geográfica y cantidad de horas dedicadas al negocio. (Cuevas, 2005) 
Se define a la microempresa como el conjunto de unidades productivas en los que laboran hasta cinco personas (seis incluido el propietario) y sólo en el caso de las manufacturas podrán ser hasta quince personas (16 incluido el dueño). Estas micro unidades productivas incluyen según la información que reporta la Encuesta Nacional de Ocupación y Empleo (ENOE, 2010), las actividades económicas que se ejercen con o sin un local y/o sin registro en alguna organización o institución del sector público.

Con base en los resultados de la ENAMIN (Encuesta Nacional de Micro-negocios, 2012) de 1998, se estimaba en nuestro país un total aproximado de 4.8 millones de micro negocios, que daban ocupación a 9.2 millones de personas. De ahí la importancia de este sector y si se piensa en los micronegocios de los mercados estos considerando el número de puestos pueden ser considerados como una unidad económica importante que representa fuente directa de ingresos para más de quinientas personas en el caso que nos ocupa.

Como se dijo anteriormente los mercados han ido desapareciendo como resultado de la llegada de supermercados con un concepto diferente y áreas de estacionamiento de la que carecen la mayor parte de ellos, pesar de ello se mantienen tampoco todos los pequeños negocios mueren. En muchas ciudades Europeas pequeños negocios han sobrevivido en la misma calle e inclusive compitiendo con supertiendas que ofrecen una gran variedad de productos y servicios (Gruenhagen and Mittelstaedt, 2001), que basan si éxito en la relación que mantienen con el cliente (Kotorov, 2003) y por un conocimiento tácito que tienen como resultado de su permanencia en el negocio.

Por otro lado a los mercados se les puede considerar como poblaciones organizacionales entendiendo como población un conjunto de organizaciones que se desempeñan en actividades parecidas, con patrones similares de utilización de recursos y resultados. Que forman nichos organizacionales considerando factores como la tecnología, estructura, productos, metas y personal específicos de una organización, sujetos a la selección o rechazo del ambiente.
La ecología organizacional, concebida por Hannan y Freeman $(1977,1989)$, rompe con el supuesto básico de la adaptación organización- entorno. Las organizaciones no se adaptan plácidamente a su entorno, sino que, por el contrario, los cambios en el entomo pueden y de hecho las hacen desaparecer. Las organizaciones que no poseen las características exigidas por el entorno serán eliminadas del mercado por un proceso de selección natural, o sea, si no se adaptan son expulsadas del mercado, y si lo intentan la inercia dificulta, retrasa e incluso imposibilita el proceso, por lo que serán eliminadas igualmente. Surge así un círculo vicioso por el que, invariablemente, desaparecerán todas aquellas organizaciones que no respondan al "ideal" organizativo que finalmente prevalecerá en el mercado.

De acuerdo con esta teoría ecológica, en el largo plazo, una vez que hayan actuado plenamente las fuerzas de la selección, existiría en cada sector — denominado nicho por los autores - una sola población de organizaciones. Es decir permanecerán las mejores y las que cambien se compartirán una población pero todas las organizaciones pertenecientes a ella se caracterizarán por tener un único estilo o forma organizativa y una dependencia común de las fuerzas del entorno (McKelvey, 1983; Hannan y Freeman, 1986; Carrol y Hannan, 1995), de modo que pertenecerán a una única configuración o tipo de organización. Es decir el cambio o la adaptación se dará en sentido dirigido. (Campos, 2000)

\section{Objetivo}

El objetivo de la presente investigación es conocer si efectivamente el "Mercado Gómez Palacio" está constituido por una población organizacional que ha sabido adaptarse a los cambios de entorno, las estrategias que se han implementado, y conocer las demandas para enfrentar nuevos cambios.

Estableciendo como hipótesis: Que las unidades económicas del mercado (micronegocios familiares) relacionadas entre ellas sobreviven como poblaciones organizacionales,

Ho. Al menos un $50 \%$ han realizado cambios para adaptarse al entorno. 
Tabla 1. Puesto Encuestados por Actividad

\begin{tabular}{lcllll}
\hline \multicolumn{1}{c}{ Comercio } & $\#$ & Servicio & $\#$ & Producción & $\#$ \\
\hline Frutas y Verduras & 1 & Florería & 9 & Artesanías & 9 \\
Piratería & 2 & Fondas & 5 & Huarachería & 1 \\
Ropa & 3 & Electrónica & 1 & Fábrica Botas & 1 \\
Hierbera & 8 & Bolero & 1 & Carnicería & 3 \\
Cremería & 2 & Cerrajería & 1 & Talabartería & 7 \\
Artesanías & 15 & Imprenta & 1 & Peletería & 1 \\
Alfarería & 5 & Relojería & 1 & & \\
Canastas & 1 & Maquinitas & 1 & & \\
Santería & 3 & Carnicería & 3 & & \\
Zapatería & 4 & & & & \\
Accesorios Celular & 1 & & & & \\
Jarcería & 1 & & & & \\
Bolsas y Mochilas & 1 & & & & \\
Marcos & 1 & & & & \\
Bisutería & 2 & & $\mathbf{2 3}$ & & \\
\hline Total & $\mathbf{5 0}$ & & & & \\
\hline
\end{tabular}

Y se pretende responder a las siguientes preguntas:

¿Por qué se han mantenido los negocios?

¿Cuáles son sus demandas para enfrentar al entorno cambiante?

\section{Método}

La investigación es descriptiva transversal y la información se obtuvo a través de un instrumento diseñado específicamente para esta investigación para reunir información en tomo al contexto del mercado, su locatarios organización y cambios que han enfrentado.

\subsection{Muestra}

La muestra fue de elección, el levantamiento de la información se levantó en una semana en junio del 2015 en base a los que quisieron completar la encuesta hasta completar una muestra de 92 puestos se pretenden representativos de las diferentes actividades que se realizan en el mercado y que se distribuye según la siguiente tabla en función de giros comercial, de producción y servicios. (Ver Tabla 1)

\subsection{Instrumento}

El instrumento de medición utilizado fue una entrevista realizada a cada uno de los locatarios de manera individual, dicha entrevista constaba de 41 preguntas de las cuales 12 estaban relacionadas con el ámbito sociodemográfico y persona del entrevistado, 19 preguntas respecto al negocio y 10 preguntas relacionadas con los retos y necesidades que han enfrentado y la manera en que se han adaptado a los cambios y amenazas que ofrece el entorno.

\section{Resultados}

Descripción de la muestra. Con base en el presente estudio, se observó que más de la mitad de los locatarios se encuentra en un rango de edad que va de los 41 a 60 años, seguido de los de edades entre 21 a 40 años, los cuales representan una cuarta parte del total de encuestados, y al final los de edades de 61 años en adelante, siendo estos la sexta parte del total de la muestra, más del $60 \%$ de los locatarios son casados, mientras que un $25 \%$ son solteros y los restantes se encuentran divorciados o viudos. 
Tabla 2. Edad de locatarios

\begin{tabular}{lcc}
\hline \multicolumn{1}{c}{ Edad } & $\#$ & $\%$ \\
\hline 0-20 años & 3 & $3 \%$ \\
21-40 años & 24 & $26 \%$ \\
$41-60$ años & 50 & $54 \%$ \\
61 años o mas & 15 & $16 \%$ \\
\hline Total & $\mathbf{9 2}$ & $\mathbf{1 0 0 \%}$ \\
\hline
\end{tabular}

Resulta interesante observar que el $98 \%$ de los entrevistados viven con su familia, mientras que tan solo un $2 \%$ residen solos. Respecto al número de hijos, se observó que el $60 \%$ de los locatarios tienen de 1 a 3 hijos; el 18\% tiene de entre 4 a 6 hijos; el $4 \%$ tiene de 7 a 9; el $2 \%$ tiene más de 10 ; y finalmente el $16 \%$ no tiene hijos. El $80 \%$ de los locatarios vive en casa propia, mientras que el $20 \%$ reside en una vivienda ya sea de renta, prestada, o bien, viven con algún familiar. Concerniente a la seguridad social, podemos observar que el $38 \%$ de los locatarios que fueron parte de la muestra no tienen ningún tipo de seguridad social, mientras que un $34 \%$ está dentro del programa del Seguro Popular; el 21\% está afiliado al IMSS y un 7\% tiene ISSTE.

Las Unidades Económicas. En mercado Gómez Palacio se realizan actividades muy diversas comerciales (56\%), de servicios (29\%) e inclusive de producción (Tabla 1, 3, Figura 1) siendo la actividad comercial la que predomina, esta actividad tiene una gran variedad de giros, y se puede apreciar que hay algunos que no solo comercializan sino que también producen sus propios artículos.
Tabla 3. Actividad de los negocios del mercado

\begin{tabular}{lcc}
\hline \multicolumn{1}{c}{ Giro } & Unidades & Porcentaje \\
\hline Comercio & 50 & $54 \%$ \\
Servicio & 23 & $25 \%$ \\
Producción & 19 & $21 \%$ \\
\hline & 92 & $100 \%$
\end{tabular}

Figura 1. Giro de los negocios Encuestados

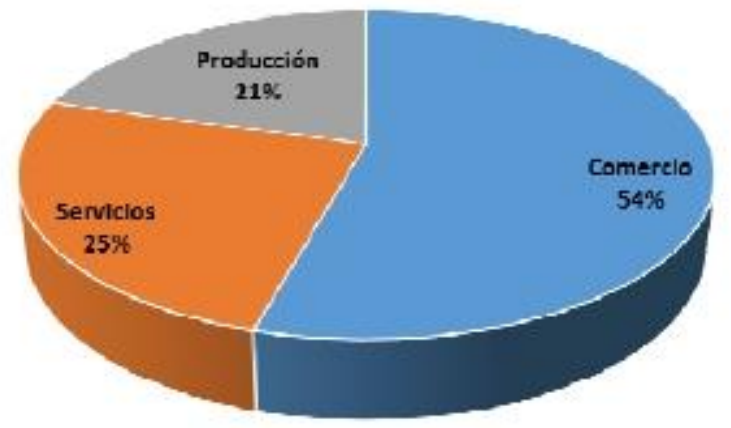

Los horarios de los locatarios y los trabajadores son variantes; algunos de estos comienzan sus labores a las 08:00 horas, algunos otros a las 09:00 horas, y una pequeña parte lo hace a partir de las 10:00 horas, aunque todos realizan el cierre a las 20:00 horas.

En cuanto al transporte de las mercancías, más del $50 \%$ de los locatarios van hasta el establecimiento de los proveedores para adquirirlas, justificando que de esta manera ahorran en costos, lo que les permite ofrecer su producto a precios más competitivos, un $37 \%$ tiene proveedores foráneos y por paquetería lo recibe un $9 \%$. El almacenaje de los productos y mercancía de los locatarios se hace, generalmente, en el mismo local, ya que no se tiene un gran excedente, siendo los principales aspectos que los locatarios toman en cuenta al momento de elegir a sus proveedores la calidad y el precio. EI precio de los productos es con base en sus costos, agregando un porcentaje de utilidad, tomando en cuenta siempre los precios de la competencia. 
Es muy reducido el número de entrevistados propietarios de locales dentro del mercado que lleva un orden contable respecto a su negocio, pues la mayoría no tiene registrados tanto sus gastos como sus ingresos, lo que representa una desventaja en la planeación, pues no tienen la posibilidad de cuantificar sus ingresos y sus gastos, pues no reciben notas de compra ni registros de las ventas, excepto por algunos que lo hacen por medio de apuntes para conocer sus ganancias aproximadas.

La mayor parte afirma que las utilidades que obtienen les son suficientes para cubrir cómodamente sus necesidades, los cuales se distribuyen de la siguiente forma: el $35 \%$ percibe ingresos menores a $\$ 5,000.00$ mensuales; el $36 \%$ obtiene ingresos que oscilan entre $\$ 5,000.00$ y $\$ 10,000.00$ mensuales; el $14 \%$ recibe entre $\$ 10,000.00$ y $\$ 20,000.00$; mientras que el $15 \%$ afirma que registra más de $\$ 20,000.00$, todos estos también de manera mensual.

La mayoría de los entrevistados respondieron que no llevan a cabo ninguna estrategia de promoción y publicidad de su mercancía, sino que esperan sólo a que los clientes lleguen a adquirir su producto. Algunos mencionan que al exhibir su mercancía están haciendo promoción de ésta, mientras que sólo unos pocos dicen entregar volantes para promocionar sus productos $y / 0$ servicios, y una mínima cantidad dice estar publicitada en la "sección amarilla".

Figura 2. Promoción de la Mercancía

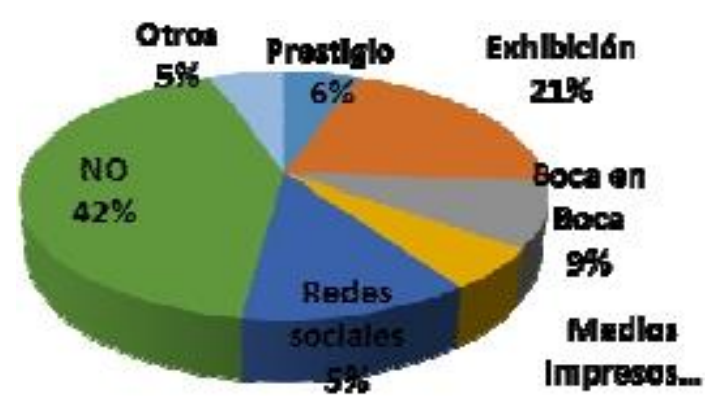

Fuente de las figuras: Elaboración propia
Respecto a la selección de los empleados, del total de los locatarios que dicen ocuparlos, (8.7\%) lo elige por tratarse de una persona a la cual conoce; lo hace debido a que le tiene confianza, lo elige por ser familiar; y en menor proporción los selecciona por tener conocimiento del negocio mediante evaluación de solicitudes de empleo. En cuanto a la jornada laboral, la mayoría trabaja más de 10 horas al día, por lo que es una labor que demanda mucho tiempo.

Las relaciones (Figura 3) dentro del mercado, son consideradas como buenas o muy buenas en general en un $77 \%$. Finalmente, respecto al ambiente laboral, podemos observar en la siguiente gráfica que se percibe un ambiente sano de trabajo por la mayor parte de los locatarios del mercado. (69\%). (Figura 4) Es decir es un lugar con buenas relaciones y ambiente de trabajo en el mayor de los casos solamente dos entrevistados lo reportaron como malo.

Figura 4. Ambiente Laboral

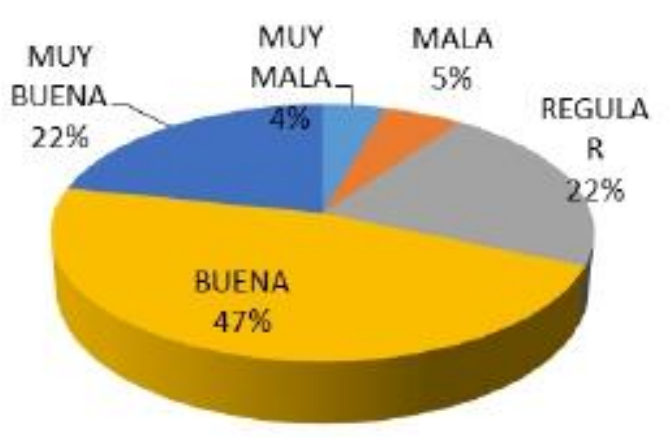

Figura 6. Relación con otros locatarios

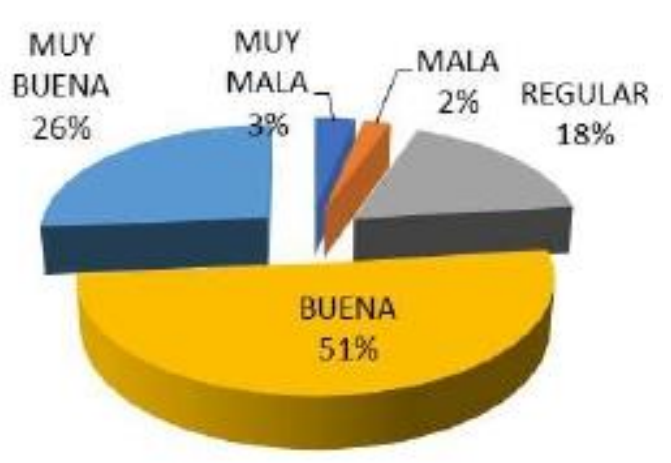




\section{Poblaciones organizacionales}

El hecho de que las actividades comerciales, de servicios y de producción nos indica que estas unidades económicas con giros semejantes están relacionadas entre sí (Figura 7).

Figura 7. Familiares en el Mercado

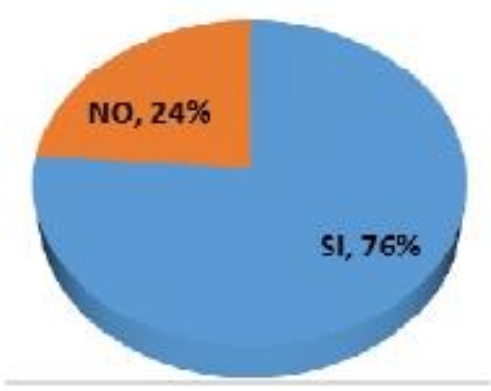

De nuestra muestra consultada, poco más de la mitad ha afirmado que sus ventas se han disminuido debido a la llegada de los supermercados (Figura 8, Tabla 4) y que provocaron el cierre de muchos de los locales que se dedicaban a frutas y verduras, abarrotes y demás suministros alimenticios, los que afirmaron que no se han visto afectados es porque aún no venden lo que ellos tiene a la venta como por ejemplo recuerdos de típicos de Durango o son negocios nuevos.

Tabla 4. Se han visto afectadas sus utilidades por los supermercados

\begin{tabular}{ccc}
\hline & Puestos & $\%$ \\
\hline $\mathrm{Si}$ & 54 & $59 \%$ \\
No & 38 & $41 \%$ \\
\hline
\end{tabular}

Figura 8. Locatarios afectados por los Supermercados

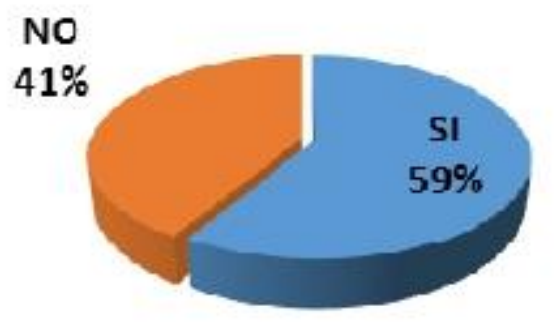

En los últimos años las ventas han bajado así lo percibe el $41 \%$ de los locatarios del mercado, mencionan "que las ventas del mercado bajaron en la década de los 70 cuando soriana llegó a Durango" aunque el $24 \%$ perciben que las ventas del mercado han mejorado (principalmente los vendedores de artesanías son los que mencionan que las ventas han mejorado) y el resto considera que siguen igual, pero no es la competencia interna lo que los afecta el $71 \%$ de los locatarios del mercado manifiestan que no les afecta la competencia dentro del mercado, (los locatarios comentan que en vez de afectarles les beneficia), consideran que la competencia que les afecta es la externa aunque hay un $29 \%$ restante si le afecta la competencia dentro del mercado. (La competencia de afuera es la que les afecta)

Los micronegocios han enfrentado el cambio adaptándose a nuevas circunstancias muchos de ellos cambiando el giro original de su negocio has dos veces en los últimos 20 años. (Figura 9)

Figura 9. Han cambiado de giro

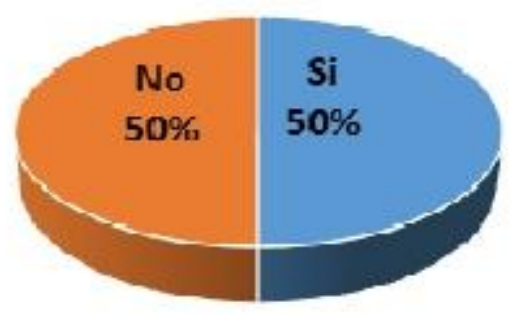

\section{Los puestos del mercado logran su permanencia,}

gracias a ser constantes su negocio se ha podido mantener, dar un buen trato a sus clientes y mencionan que dar sus artículos a un buen precio también les ha permitido mantenerse en el negocio. Entre otros aspectos que mencionan los locatarios para que siga manteniéndose su negocio están: La buena administración, el hecho de echarle ganas, adaptarse al cambio, la mercancía de buena calidad, que ya tienen su clientela, por temporadas altas, buenas ventas, entre otras cosas. (Figura 10) 
Figura 10. Causas de la estabilidad del negocio

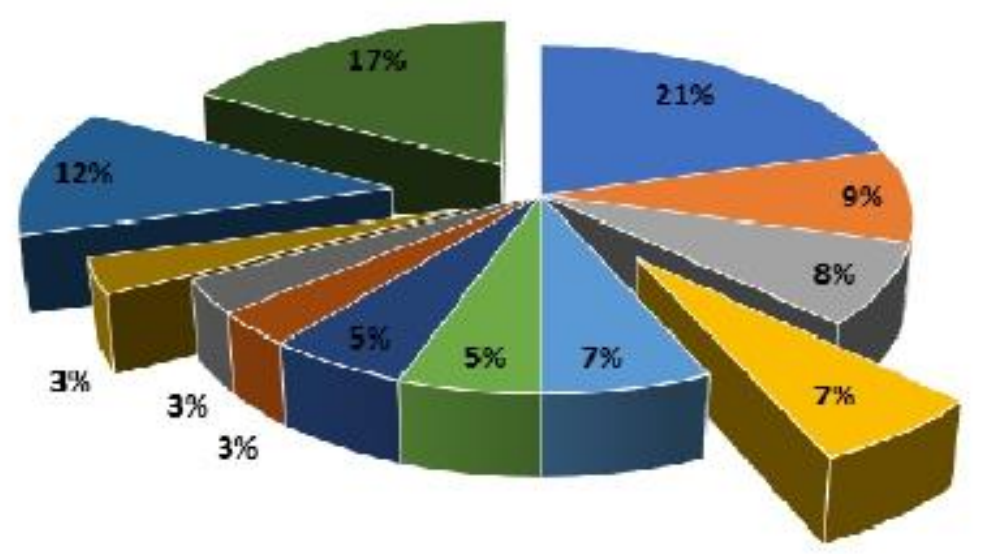
- a) Constancla
- b) Buen trato al cllente
II C) Buenos preclos
- d) Le echamos ganas
- e) Buena Ad ministración
- f)Adap tarse al cambio
- в) Mercancia de buena ca lidad - h) Ya tiene su clientela
- i) Temporadas altas

- J) Buenas ventas

- k) otros

Figura 11. Demandas de los locatarios

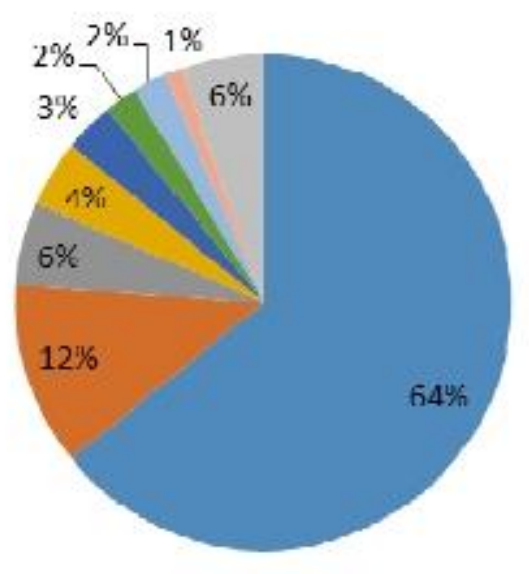

\author{
Más publicidad \\ - Agregar estacionamiento \\ a Dar mís mantenimiento al \\ mercado \\ - Mejorar el trato a los \\ clienles \\ - I rabajo en equipo \\ administr ación eliciente
}


Sucesión. Dentro de nuestra muestra se preguntó si habían pensado quien se quedará a cargo del negocio cuando ellos se retiraran y el $71 \%$ contesto que sí y dentro de otra pregunta que decía que si había interés por parte de sus hijos de continuar el negocio fue positivo el $60 \%$, esta comparación nos muestra que la tendencia de que el negocio sea familiar y lo manejen los hijos es casi un $85 \%$ el otro $15 \%$ si pensó que si quisieran que se quedara con su negocio, pero no hay interés por parte de los hijos.

Carencias detectadas. De acuerdo a las respuestas de los locatarios un factor del cual carecen en gran medida es la Publicidad, esto trae por consecuencia diversos problemas, como el hecho de quienes asisten a Durango como turistas simplemente desconocen que hay un mercado municipal y que en él pueden encontrar artículos de su interés como lo son los "recuerditos" de su visita a la ciudad.

\section{(Ver Figura 11)}

Con un porcentaje del $64 \%$ la publicidad se posiciona en el primer lugar como propuesta para incrementar las ventas de todo el mercado, darlo a conocer no sólo a turistas, sino también a quienes habitan en la ciudad, cuya concurrencia ha disminuido con el paso de los años; según los locatarios el apoyo por parte del Gobierno del Estado es vital, particularmente de la Secretaría de Turismo, quienes pueden lograr un mayor alcance de información.

Otro factor que los locatarios consideran de gran importancia es la integración de un estacionamiento para el mercado ya que no cuentan con uno; han observado las dificultades de los proveedores para hacer la entrega de mercancía así como de los mismos clientes para encontrar un lugar disponible y hacer sus compras, este factor se presenta con un $12 \%$ de las respuestas.

El mantenimiento al mercado se presenta con un $5 \%$, los locatarios consideran que hacerlo más atractivo puede ser una buena alternativa para incrementar sus ventas; con el mismo porcentaje se presenta el que no consideran que el mercado necesita algo o que simplemente no haya nada por hacer.
Con un $4 \%$ se presenta la mejora en el trato a los clientes, mientras que despejar las puertas y pasillos así como el tener una administración eficiente se presenta con un $2 \%$, finalmente con un $1 \%$ hay quienes consideran que un cambio de giro en los locales puede resultar más atractivo ya que habrá más variedad de productos y servicios.

\section{Conclusiones}

En relación a la hipótesis y a las preguntas de investigación tenemos:

1. En base a la información generada no se puede negar que el mercado Gómez Palacio se puede considerar uno población organizacional que ha sabido adaptarse a los cambios del entorno un $50 \%$ ha cambiado de giro y los que permanecen es porque han cumplido con las exigencias del entorno. (Figura 8, 9, 10). Aceptándose la hipótesis.

2. Los micronegocios han sobrevivido porque han respondido al cambio de diversas formas, cambiando de giro y desde el punto de vista de los locatarios por lo que otros autores reportan su cercanía al cliente, constancia, esfuerzo entre otras. (Figura 10)

3. Las demandas más sentidas son publicidad, estacionamiento y mantenimiento al Mercado (Figura11)

La imagen de un mercado es el producto o servicio real que se vende. Se basa en obtener el producto correcto y en conseguir transmitir dicha imagen para que sea percibida. Para sobrevivir el mercado municipal necesita apostar por un proceso evolutivo que le ayude a avanzar en nuevos proyectos sin llegar a perder sus tradiciones que lo caracterizan. Los mercados han de ser capaces de rentabilizar hacia el futuro la buena imagen tradicional que siempre han tenido como formato de proximidad de cara a los consumidores y que se refleja en factores, tales como su identificación como garantía de calidad, su trato personalizado y su contacto directo con el entorno urbano, es decir deben ser capaces de adaptarse al medio. 


\section{Agradecimientos}

Se reconoce la participación y colaboración para la realización del presente proyecto de nuestros compañeros del VII Verano de Investigación de la FECA-UJED Juvenal Casas Duarte, Carlos Fernando Alvarez Sosa, Jorge Alberto Mancinas, Edith García, Vanessa Pablos Esquivel, Diana Karina Soto Martínez, Carlos Ignacio Díaz Zamora y Luis Ávila Flores y de los recursos para apoyo de esta investigación de Programa Integral de Fortalecimiento Institucional PIFI-2015.

\section{Bibliografía}

Campos, S., Roberto, C., Duran, C., Fernández, O.H (2000). Un nuevo enfoque para el análisis de las organizaciones. La Ecología Organizacional. Faces, 6(9), 9-22.

Carrol y Hannan, M. T. (1995). Organizations in Industry. Strategy, Structure.

and Selection, New York, Oxford: Oxford University Press.

Cuevas, E., Contreras, A. and González, A. (2005). Micronegocios y autoempleo en México. Un análisis empírico con datos de la Encuesta Nacional de Micronegocios. Carta Económica Regional, núm. 94.

Encuesta Nacional de Ocupación y Empleo (ENOE, 2010)

Encuesta Nacional de Micro-negocios, 2012

Gruenhagen, M. and Mittelstaedt, R. (2001). The impact of store hours and redistributive income effects on the retail industry: some projections for Germany. The International Review of Retail, 11(1), 49-62.

Hannan, M. T. y Freeman, J. (1977). The Population Ecology of Organizations. American Journal of Sociology, 82, 929-964.

Hannan, M.T. y Freeman, J. (1984), "Structural Inertia and Organizational Change". American Sociological Review, 49, 149-164.
Kotorov, R. (2003), "Customer relationship management strategic lessons and future directions", Business Process Management Journal, 9(5), 566-571.

Lastra, G. (2013). "La tradición del Mercado Gómez Palacio". [En línea] Milenio. Disponible en:

http://www.milenio.com/laguna/tradicion-MercadoGomez-Palacio_0_161984022.html [Acceso 23 Ago. 2016].

McKelvey, B. y Aldrich, H. (1983). Natural Selection and Applied Organizational Science. Adminsitrative Science Quaterly, 28(1), 101-128.

Rosales, G. (2013). "Mercado Gómez Palacio, identidad del 450 de Durango". [En línea] Periódico Contexto de Durango | Noticias minuto a minuto en Durango. Disponible en:

http://contextodedurango.com.mx/noticias/2013/09/07/ mercado-gomez-palacio-identidad-del-450-de-durango/ [Acceso 23 jun. 2016]. 